Original Article

\title{
Paravertebral Block as a Promising Analgesic Modality for Managing Post-Thoracotomy Pain
}

Teruya Komatsu, MD, Terumasa Sowa, MD, Koji Takahashi, MD, and Takuji Fujinaga, MD, PhD

\begin{abstract}
Purpose: Analgesia following thoracotomy is routinely accomplished using epidural blockage performed by anesthesiologists. More effective pain control can be achieved with continuous paravertebral blockage performed by thoracic surgeons. We aimed to retrospectively analyze the efficacy of paravertebral blockage for managing post-thoracotomy pain. Methods: The study included 125 patients who underwent continuous paravertebral blockage for the following types of thoracic surgery: video-assisted thoracoscopic surgery $(n=87)$, anterior axillary thoracotomy $(n=21)$, posterolateral thoracotomy $(n=16)$, and median sternotomy with additional left thoracotomy $(n=1)$. We retrospectively evaluated the analgesic effects of continuous paravertebral blockage by assessing whether a good cough effort could be performed and whether an additional painkiller was given as a rescue medication.

Results: About 115 patients could perform a good cough effort to expectorate sputum immediately after extubation in the operating room. Six patients tolerated postoperative pain well without any oral or rectal non-steroidal anti-inflammatory drugs (NSAIDs). For 97 patients postoperative NSAIDs could control thoracotomy pain well. Twenty-two patients were given an additional painkiller stronger than NSAIDs. Three patients complained of nausea postoperatively.

Conclusion: Adequate post-thoracotomy pain control was accomplished by continuous paravertebral blockage, with few complications.
\end{abstract}

Keywords: paravertebral block (PVB), epidural block, continuous intercostal nerve block, thoracotomy

\section{Introduction}

Thoracotomy often causes severe acute postoperative pain, which leads to a high incidence of pulmonary complications. ${ }^{1,2)}$ Unless controlled sufficiently, impaired

Department of General Thoracic Surgery, Nagara Medical Center, Gifu, Gifu, Japan

Received: April 5, 2012; Accepted: September 24, 2012

Corresponding author: Teruya Komatsu, MD. Department of General Thoracic Surgery, Nagara Medical Centre, 1300-7 Nagara, Gifu, Gifu, Japan

Email: tk.thoracic@gmail.com

(C)2014 The Editorial Committee of Annals of Thoracic and Cardiovascular Surgery. All rights reserved. pulmonary function can deteriorate. ${ }^{3)}$ Effective pain control following thoracotomy should be a prerequisite for uneventful postoperative recovery.

Thoracic epidural analgesia is considered the golden standard for pain control following thoracotomy. ${ }^{4-6)}$ However, there are some complications and contraindications associated with the epidural techniques. ${ }^{6-8)}$

We have introduced continuous infusion of local anesthetics through an indwelling epidural catheter placed in the extrapleural space by surgeons. This technique was originally described and updated by Sabanathan and colleagues. ${ }^{9,10)}$ Most anesthesiologists, as well as general thoracic surgeons, in Japan are not familiar with this method of pain control. The current study is based on the 
retrospective analysis of our results using the updated Sabanathan's technique.

There are various terminologies for this anesthetic technique, for example, paravertebral block (PVB), continuous intercostal nerve block, extrapleural intercostal nerve block, extrapleural PVB, and retropleural analgesia. ${ }^{8,11)}$ Eng and colleagues radiologically showed that the site of action of local anesthetic administered via an extrapleural intercostal catheter is primarily via the paravertebral space. ${ }^{12)}$ Therefore, in our report, the term PVB is used for continuous intercostal nerve blockage.

\section{Materials and Methods}

About 125 patients ( 79 men and 46 women), with a mean age of 68 years (range, 32 to 88 years), underwent PVB catheter insertion. All of these patients underwent operations for pulmonary resection. Eighty-seven (male/ female, 49/38) patients underwent video-assisted thoracoscopic surgery (VATS), 21 (male/female, 17/4), cases through anterior axillary thoracotomy (AAT); 16 (male/ female, 12/4), posterolateral thoracotomy (PLT); and 1, median sternotomy with additional left thoracotomy (Med). Our VATS approach involves 3 incisions. The largest is the utility incision of about $5 \mathrm{~cm}$, which is placed over the 4th or 5th interspace; a rib spreader is not usually applied. The other 2 incisions are about 10-20 mm and are made for the thoracoscopic ports.

\section{Technique of PVB catheter insertion}

Before closing the thoracotomy, a PVB catheter was inserted. By peeling back the parietal pleura medially as far as the neck of the ribs to expose the intercostal nerves starting at the thoracotomy opening, an extrapleural pocket is developed posteriorly for 2 to 3 interspaces above and below the level of the thoracotomy. An 18-gauge epidural catheter is then placed percutaneously into this extrapleural space under direct visualization. The final position of the catheter is with the lower portion in the inferior part of the pocket and the tip in the superior portion of the pocket (Fig. 1). ${ }^{9,10,13)}$ Then, following lung re-inflation, we administered $0.5 \%$ bupivacaine hydrochloride (Marcain Plain; Astra, Osaka, Japan) intermittently through the indwelling epidural catheter placed in the above-mentioned manner so that a total dosage of $20 \mathrm{~mL}$ is administered at the completion of chest closure. After the patient is transferred back to the recovery room, continuous infusion of $1 \%$ lidocaine at $6 \mathrm{~mL} / \mathrm{h}$ (Xylocaine; Astra, Osaka, Japan) is initiated through an

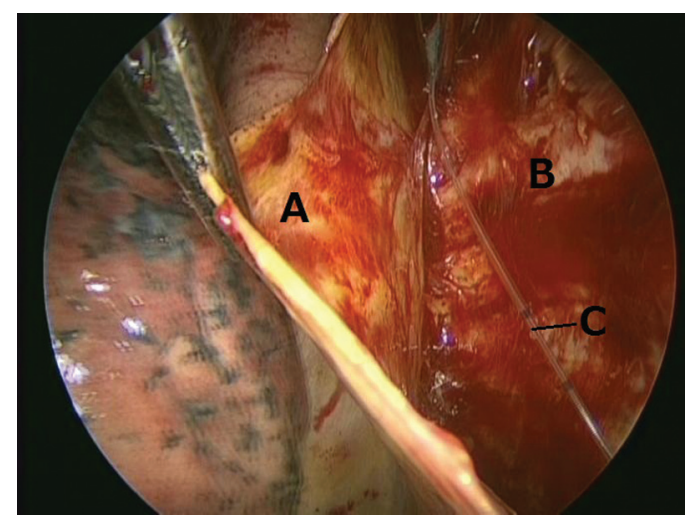

Fig. 1 Paravertebral block (PVB) catheter placed under the direct vision of the surgeon. (A) parietal pleura peeled back medially. (B) chest wall. (C) PVB catheter. The tip of the catheter is guided towards the superior portion of the pocket (paravertebral space).

infusion pump.

Postoperatively, patients are strongly encouraged to ask for a painkiller if needed, without hesitation, to allow for a good cough effort and rigorous physiotherapy. The first choice of analgesic for the patients in our analysis was a non-steroidal anti-inflammatory drug (NSAID) (Loxonin: loxoprofen sodium hydrate; Daiichi-Sankyo, Tokyo, Japan). In case of analgesic failure with NSAIDs, Pentagin (pentazocine hydrochloride; DaiichiSankyo, Tokyo, Japan) was administered intramuscularly/ intravenously as a rescue medication. We measured the analgesic efficacy of PVB in terms of whether patients could perform a good enough cough to clear the airway and whether or not the rescue analgesic was given to supplement the efficacy of NSAIDs. Furthermore, the side effects related to PVB were also investigated.

\section{Results}

About 115 (92.4\%) could perform a good cough soon after extubation in the operating room and could expectorate sputum very well. Postoperatively, all patients were advised not to attempt to endure intractable pain and to ask for an additional painkiller without hesitation. Six patients did not need any pain medication. Postthoracotomy pain in 97 patients (77.6\%) was effectively controlled with oral or rectal NSAIDs, without an additional stronger painkiller. Twenty-two patients (17.6\%; 14 VATS, 2 AAT, 5 PLT, 1 Med) requested an additional painkiller as a rescue medication (pentazocine hydrochloride) (Fig. 2). There were almost no complications related 


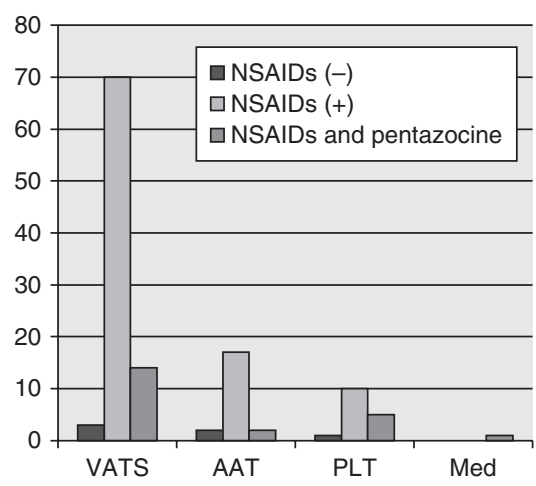

Fig. 2 Postoperative pain medication. Most of the patients could tolerate postoperative pain well.

either to the insertion procedure or to the infusion of local anesthetics, except in 3 patients $(2.4 \%)$ who had nausea postoperatively. All of the patients were cooperative for physiotherapy and no pain-related complication was noted.

\section{Discussion}

The principal weakness of our analysis is that it is neither randomized nor comparative, but retrospective. Thoracic epidural blockage had not been used for pain control before the introduction of PVB at our institution. This made it impossible for us to compare analgesic efficacy between conventional thoracic epidural blockage and PVB. Therefore, our primary goal in this report is to introduce the effectiveness of PVB for thoracotomy pain with reference to the relevant literature.

Epidural anesthesia seems to be the method of choice for thoracotomy due to its well-known effective analgesia. However, the epidural technique is also known for its adverse effects, which include urinary retention (42\%), nausea $(22 \%)$, itching (22\%), hypotension (3\%), and respiratory depression $(0.07 \%)$. Prolonged operative time and technical failure are often additional drawbacks $(8 \%)^{14)}$. The epidural technique is also contraindicated in septic conditions, coagulation ailments, pre-existing neurological disorders, and cases of difficult vertebral anatomy $^{8}$. Even though thoracic epidural analgesia is still more often employed for thoracotomy pain, multiple prospective randomized controlled studies have shown that the use of an indwelling extrapleural catheter for PVB is an effective way of decreasing postoperative pain and narcotic requirements. ${ }^{2,8,9,15)}$ According to the prospective, randomized, blinded study comparing the efficacy of the PVB and thoracic epidural techniques by Casati, et al. there was not a significant difference of rescue morphine administration (PVB group: 23\%, epidural group: 19\%; $\mathrm{p}=0.99$ ). And they suggest the clinical bottom line that PVB can be at least as effective as epidural methods. ${ }^{16)}$ However, it is not yet clear whether PVB or epidural analgesia is better for post-thoracotomy pain.2)

Recently, there has been a resurgence of PVB in the field of general thoracic surgery due to its analgesic efficacy and low side-effect profile. ${ }^{8,17)}$ Sabanathan and colleagues reported and updated this technique, which has been introduced in our thoracic team. ${ }^{9,10)}$

The paravertebral spread of local anesthetic that is continuously infused postoperatively is believed to block intercostal nerves, their collateral branches and posterior primary rami, and the thoracic sympathetic chain. ${ }^{10,12,18)}$ Blocking the posterior primary rami could alleviate backache caused by straining of vertebral muscles and ligaments of the costovertebral and costotransverse joints. Unilateral sympathetic block could have a positive influence on the endocrine-metabolic response to operation without the hypotension associated with bilateral sympathetic block induced by epidural block. ${ }^{19)}$

Our results show that postoperative pain management with thoracic PVB is very effective. There were no pain-related postoperative complications such as atelectasis and pneumonia, which can be explained by the fact that most of our patients (92.4\%) were compliant with coughing and deep breathing. However, 22 patients $(17.6 \%)$ asked for an additional painkiller (pentazocine hydrochloride). This result indicates that we could have performed better analgesia. In 18 out of these 22 patients, continuously infused local anesthetic may not have been adequately contained in the paravertebral space, because the parietal pleura was torn or penetrated during the creation of the paravertebral space. Therefore, the success rate of thoracic PVB greatly depends on intact pleura for better containment of local anesthetic. ${ }^{9)}$

An extended operative time and technical failure in epidural placement are both less likely in PVB, since the catheter for PVB is inserted under direct vision by the surgeon in less than 5 min during chest closure. ${ }^{8,20)}$ However, in some thoracic cases that necessitate resection of the pleura responsible for the paravertebral space, epidural block should be selected.

There are several medications that can be administered into the paravertebral space; however, neither the best drug nor the best concentration has been clarified so far. ${ }^{8)}$ According to the literature regarding PVB, bupivacaine is one of the most commonly used drugs. ${ }^{2,9,12,19,20)}$ In our 
study, bupivacaine was administered for intermittent dosing until the completion of chest closure because of its long half-life, and lidocaine was administered for postoperative continuous infusion because of its shorter half-life. Considering the side effects of bupivacaine, which are central nervous toxicity, severe myocardial suppression, and prolonged asystole, lidocaine might have been an alternative choice for intermittent dosing in our study. ${ }^{13,21)}$

\section{Conclusion}

The analgesic superiority of PVB over conventional epidural blockage is not elucidated in our report. However, due to its technical simplicity, low incidence of side effects, and analgesic effectiveness, PVB can be recommended for pain control following thoracotomy.

\section{Disclosure Statement}

The authors have no conflict of interest in the publication of this manuscript.

\section{References}

1) Smith RA. Letter: Pain relief after thoracotomy. Lancet 1976; 1: 815-6.

2) Kotzé A, Scally A, Howell S. Efficacy and safety of different techniques of paravertebral block for analgesia after thoracotomy: a systematic review and metaregression. Br J Anaesth 2009; 103: 626-36.

3) Richardson J, Sabanathan S, Shah R. Post-thoracotomy spirometric lung function: the effect of analgesia. A review. J Cardiovasc Surg (Torino) 1999; 40: 445-56.

4) Joshi GP, Bonnet F, Shah R, et al. A systematic review of randomized trials evaluating regional techniques for postthoracotomy analgesia. Anesth Analg 2008; 107: 1026-40.

5) Gerner P. Post-thoracotomy pain management problems. Anesthesiol Clin 2008; 26: 355-67.

6) Messina M, Boroli F, Landoni G, et al. A comparison of epidural vs. paravertebral blockade in thoracic surgery. Minerva Anestesiol 2009; 75: 616-21.

7) De Cosmo G, Aceto P, Gualtieri E, et al. Analgesia in thoracic surgery: review. Minerva Anestesiol 2009; 75: 393-400.

8) Davies RG, Myles PS, Graham JM. A comparison of the analgesic efficacy and side-effects of paravertebral vs epidural blockade for thoracotomy-a systematic review and meta-analysis of randomized trials. Br J Anaesth 2006; 96: 418-26.

9) Sabanathan S, Smith PJ, Pradhan GN, et al. Continuous intercostal nerve block for pain relief after thoracotomy. Ann Thorac Surg 1988; 46: 425-6.

10) Sabanathan S, Richardson J, Shah R. 1988: Continuous intercostal nerve block for pain relief after thoracotomy. Updated in 1995. Ann Thorac Surg 1995; 59: 1261-3.

11) Karmakar MM, Critchley L. Continuous extrapleural intercostal nerve block for post thoracotomy analgesia in children. Anaesth Intensive Care 1998; 26: 115-6.

12) Eng J, Sabanathan S. Site of action of continuous extrapleural intercostal nerve block. Ann Thorac Surg 1991; 51: 387-9.

13) Watson DS, Panian S, Kendall V, et al. Pain control after thoracotomy: bupivacaine versus lidocaine in continuous extrapleural intercostal nerve blockade. Ann Thorac Surg 1999; 67: 825-8; discussion 828-9.

14) Detterbeck FC. Efficacy of methods of intercostal nerve blockade for pain relief after thoracotomy. Ann Thorac Surg 2005; 80: 1550-9.

15) Sullivan E, Grannis FW, Ferrell B, et al. Continuous extrapleural intercostal nerve block with continuous infusion of lidocaine after thoracotomy. A descriptive pilot study. Chest 1995; 108: 1718-23.

16) Casati A, Alessandrini P, Nuzzi M, et al. A prospective, randomized, blinded comparison between continuous thoracic paravertebral and epidural infusion of $0.2 \%$ ropivacaine after lung resection surgery. Eur J Anaesthesiol 2006; 23: 999-1004.

17) Pipanmekaporn T, Saeteng S. The use of continuous thoracic paravertebral nerve block under direct vision for postoperative pain management in thoracic surgery. J Med Assoc Thai 2012; 95: 191-7.

18) Sabanathan S, Mearns AJ, Bickford Smith PJ, et al. Efficacy of continuous extrapleural intercostal nerve block on post-thoracotomy pain and pulmonary mechanics. Br J Surg 1990; 77: 221-5.

19) Richardson J, Sabanathan S, Eng J, et al. Continuous intercostal nerve block versus epidural morphine for postthoracotomy analgesia. Ann Thorac Surg 1993; 55: 377-80.

20) Luketich JD, Land SR, Sullivan EA, et al. Thoracic epidural versus intercostal nerve catheter plus patient-controlled analgesia: a randomized study. Ann Thorac Surg 2005; 79: 1845-9; discussion 1849-50.

21) el-Baz N, Faber LP, Ivankovich AD. Intrapleural infusion of local anesthetic: a word of caution. Anesthesiology 1988; 68: 809-10. 